\title{
Band-gap structure in two- and three-dimensional cellular locally resonant materials
}

\author{
Marco Moscatelli ${ }^{1}$, Raffaele Ardito ${ }^{1}$, Larissa Driemeier ${ }^{2}$, Claudia Comi ${ }^{1, *}$ \\ ${ }^{1}$ Department of Civil and Environmental Engineering, Politecnico di Milano \\ Piazza Leonardo da Vinci 32, 20133 Milan, Italy \\ e-mail: raffaele.ardito@polimi.it,claudia.comi@polimi.it, \\ marco1.moscatelli@mail.polimi.it \\ ${ }^{2}$ Department of Mechatronics, University of São Paulo \\ São Paulo, Brazil \\ e-mail:driemeie@usp.br \\ ${ }^{*}$ Corresponding author
}

\begin{abstract}
Wave propagation in periodic locally resonant materials (LRM) is characterized by the presence of band-gaps occurring at relatively low frequencies which can be exploited for vibration attenuation or impact absorption. In this work we consider the dispersion properties of three-components cellular LRM endowed with 2D (cylindrical) or 3D periodicity. Modal analyses on the unit cell, with Bloch-Floquet periodicity conditions, are performed by the finite element methods. For cylindrical LRM, the limits of plane analyses, which consider uncoupled in-plane and out-of-plane modes, are highlighted. Several different geometries for the 3D unit cells are also proposed and their influence on the band-gap width is studied.
\end{abstract}

Keywords: metamaterial, dispersion spectrum, locally resonant, impact absorption

\section{Introduction}

Locally resonant materials (LRM) are periodic media, widely studied in view of their peculiar properties with respect to wave propagation [1]. Such materials belong to the class of metamaterials, i.e. engineered materials characterized by effective properties that cannot be found or are hardly found in natural materials. The possible applications of metamaterials are 
numerous and widespread: acoustic isolation, noise suppression, vibration attenuation, acoustic waveguides, acoustic super-lenses, negative refraction, acoustic cloaking, energy harvesting, see the review papers [2, 3, 4, 5]. Many applications harness the fact that the dispersion spectrum of metamaterials may exhibit band-gaps, i.e. frequency ranges in which wave propagation is prohibited, that depend on the vibrational features of its components. The mechanism behind wide $[6,7,8]$ and tunable $[9,10,11]$ band-gaps has been thoroughly studied, both numerically and experimentally; to improve the wave filtering performances, some researchers have proposed the application of topological optimization technique $[12,13]$.

At difference with respect to the Bragg scattering mechanism, commonly described for the so-called phononic crystals [14, 15], local resonance allows the achievement of band-gaps with a central frequency which is well below the frequency of the wave associated to the cell dimension of the periodic arrangement. Such a behavior has been interpreted as a "negative mass" effect that can be fruitfully exploited to get wave filters at relatively low frequency $[16,17,18,19]$.

In most cases, the effect of local resonance is obtained by the adoption of three-phase materials, e.g. high density inclusions with an elastic coating, embedded in a rigid matrix [20]. Such a scheme has been adopted for the mitigation of blast and impact effect, with encouraging results: the $\mathrm{Au}$ thors of [21] have shown the results of a parametric study on the basis of a mathematical model; a practical implementation of such a concept can be found in [22], that contains also an experimental validation. In the framework of impact absorbers for crash-worthiness of lightweight cars, Comi and Driemeier [23, 24] have proposed the adoption of a LRM with a different scheme: a square lattice of aluminium cells, filled by a polymeric foam, with circular lead inclusions. In that case, the study was focused on materials having two-dimensional periodicity and composed of long extruded cells that can be effectively studied by means of plane models considering propagation of pure transverse waves and planar waves separately . However, it must be noted that, in some cases, the application of $2 \mathrm{D}$ simulations may lead to misleading conclusions about the real three-dimensional case [25] and the assumption that the complete band-gap can be obtained as the intersection of those obtained with plane analyses can be questioned.

The purpose of this paper is twofold. Firstly, the attention is focussed on the LRM with 2D periodicity, proposed in [24]. Such a metamaterial is characterized by an innovative design and shows the interesting property 
of overlapping band-gap for in-plane and transverse waves. A complete 3D band-gap is thus expected, but its extension could be highly affected by the out-of-plane thickness of the real material. Therefore, the paper presents the detailed study of the dispersion diagram with the main purpose of critically examining the differences between cost-effective plane models, that consider decoupled in-plane and out-of-plane transmission modes, and more realistic full 3D models. The objective is to understand the effect of the out-of-plane dimension, with a broader perspective with respect to previous works [25]. To this purpose, considering the LRM with 2D periodicity proposed in [24], various 3D models with different out-of-plane extensions are analyzed. The $3 \mathrm{D}$ and $2 \mathrm{D}$ band structures are obtained by means of finite-element modal analysis. The dispersion analyses necessitate of specific provisions in order to introduce the Bloch-Floquet periodicity conditions on the unit cell. The comparative examination of the achieved results leads to new interesting conclusions on the interplay between in-plane and out-of-plane modes for cellular materials with limited extrusion length. Secondly, the paper tries to open new ways in order to improve the performances of the considered LRM, also in view of its practical application as shock-absorbing material. To this end, we propose, analyse and compare new designs of three-components cellular LRM with three-dimensional periodicity. The knowledge of the mechanical behavior of 3D LRM represents the basis for the development of new layouts, obtained by changing the shape of the unit cell and of the inclusions.

\section{Description of the computational technique}

LRM are represented by the periodic repetition along the lattice axes $\mathbf{e}_{1}$, $\mathbf{e}_{2}$ and $\mathbf{e}_{3}$ of a unit cell, generally composed of two or more materials (solid or, possibly, fluid). The problem of wave propagation is governed by the elasto-dynamic equations for inhomogeneous media:

$$
\begin{aligned}
\rho \frac{\partial^{2} u_{x}}{\partial t^{2}} & =\frac{\partial}{\partial x}(\lambda \nabla \cdot \mathbf{u})+\nabla \cdot\left(\mu\left(\nabla u_{x}+\frac{\partial \mathbf{u}}{\partial x}\right)\right) \\
\rho \frac{\partial^{2} u_{y}}{\partial t^{2}} & =\frac{\partial}{\partial y}(\lambda \nabla \cdot \mathbf{u})+\nabla \cdot\left(\mu\left(\nabla u_{y}+\frac{\partial \mathbf{u}}{\partial y}\right)\right) \\
\rho \frac{\partial^{2} u_{z}}{\partial t^{2}} & =\frac{\partial}{\partial z}(\lambda \nabla \cdot \mathbf{u})+\nabla \cdot\left(\mu\left(\nabla u_{z}+\frac{\partial \mathbf{u}}{\partial z}\right)\right)
\end{aligned}
$$


where $\rho$ is the material density, $\lambda$ and $\mu$ are the Lame's coefficients, $\mathbf{u}=$ $\left[u_{x}, u_{y}, u_{z}\right]$ is the displacement vector with its Cartesian components and $\nabla$ denotes the gradient vector. The material parameters are periodic in space, according to any scheme that could be used to describe crystalline materials (see e.g. [26]). In that case, the Bloch-Floquet theorem states that the solutions of the periodic dynamic equations (1) are Bloch waves of the form:

$$
\mathbf{u}(\mathbf{r}, t)=\tilde{\mathbf{u}}(\mathbf{r}) \mathrm{e}^{\mathrm{i} \mathbf{k} \cdot \mathbf{r}} \mathrm{e}^{\mathrm{i} \omega t}
$$

where $\mathbf{r}=[x, y, z]$ is the position vector, $\tilde{\mathbf{u}}(\mathbf{r})$ is a periodic function with the same periodicity of the LRM, $\mathbf{k}$ is the wave vector and $\omega$ is the angular wave frequency.

The wave propagation in the LRM can be studied by considering a unit cell, with the application of suitable boundary conditions in order to comply with the Bloch-Floquet theorem. In this study, the finite element (FE) method has been adopted in order to obtain a numerical solution, through the use of the commercial software ABAQUS. The unit cell is divided into 3D elements and the FE discretization yields the governing equation for wave propagation:

$$
\left(\mathbf{K}-\omega^{2} \mathbf{M}\right) \mathbf{w}(\mathbf{k})=\mathbf{0}
$$

$\mathbf{K}$ and $\mathbf{M}$ are the assembled global stiffness and mass matrices, $\mathbf{w}(\mathbf{k})$ is the global vector of unknowns containing the displacements components of each node. The periodic boundary conditions are enforced on corresponding surfaces, here generically denoted by $\Gamma_{n}^{+}$and $\Gamma_{n}^{-}$, where $n$ is referred to one vector in the lattice basis $\mathbf{e}_{n}$ :

$$
\mathbf{w}_{\Gamma_{n}^{+}}=\mathbf{w}_{\Gamma_{n}^{-}} \mathrm{e}^{\mathrm{i} \mathbf{k} \cdot \mathbf{e}_{n}}=\mathbf{w}_{\Gamma_{n}^{-}}\left[\cos \left(\mathbf{k} \cdot \mathbf{e}_{n}\right)+\mathrm{i} \sin \left(\mathbf{k} \cdot \mathbf{e}_{n}\right)\right]
$$

The boundary conditions can be expanded as:

$$
\begin{aligned}
& \operatorname{Re}\left(\mathbf{w}_{\Gamma_{n}^{+}}\right)=\operatorname{Re}\left(\mathbf{w}_{\Gamma_{n}^{-}}\right) \cos \left(\mathbf{k} \cdot \mathbf{e}_{n}\right)-\operatorname{Im}\left(\mathbf{w}_{\Gamma_{n}^{-}}\right) \sin \left(\mathbf{k} \cdot \mathbf{e}_{n}\right) \\
& \operatorname{Im}\left(\mathbf{w}_{\Gamma_{n}^{+}}\right)=\operatorname{Re}\left(\mathbf{w}_{\Gamma_{n}^{-}}\right) \sin \left(\mathbf{k} \cdot \mathbf{e}_{n}\right)+\operatorname{Im}\left(\mathbf{w}_{\Gamma_{n}^{-}}\right) \cos \left(\mathbf{k} \cdot \mathbf{e}_{n}\right)
\end{aligned}
$$

The solution is then obtained by considering two identical meshes, one for the real part and the other for the imaginary part of the displacement vector, linked by the boundary conditions (5). Equation (3) is solved, along with boundary conditions (5), in order to find eigenvalues $\omega^{2}$, eigenvectors $\mathbf{w}$ and the associated dispersion relation at varying $\mathbf{k}$. It is important to notice that, 
(a)
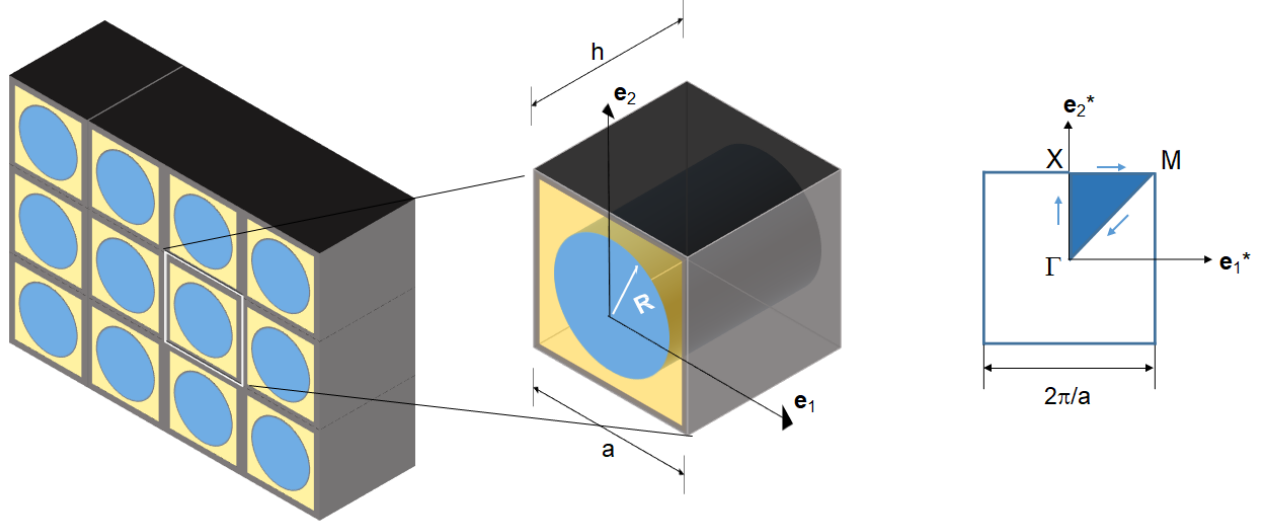

(b)
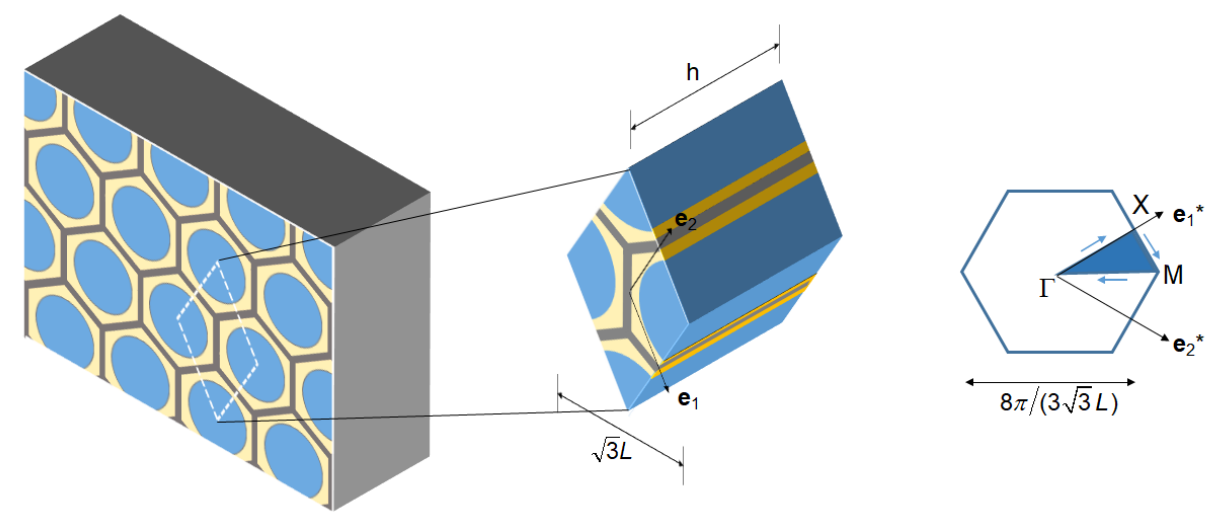

Figure 1: Cellular periodic material with cylindrical resonators, representative unit cells and first Brillouin zones. (a) Square lattice, (b) hexagonal lattice.

since the governing matrices are Hermitian, all the eigenvalues are real. Due to the symmetries of the unit cell, the description of the dynamic behavior of the LRM for all the possible wave vectors can be obtained by focusing the attention on a particular regions of the reciprocal space, called first Irreducible Brillouin Zone (IBZ). Many authors (see e.g. [27]) claim that the essential features of the dispersion diagram can be determined focusing the attention on the $\mathbf{k}$ vectors along the boundary of the first IBZ, following the paths indicated in the next Sections. 


\section{Band-gap in cylindrical locally resonant materials}

Consider a three-component composite material consisting of a cellular periodic metallic frame, filled by a soft light material with heavy inclusions of cylindrical shape (see Figure 1). We will refer to this 2D-periodic material as "cylindrical" locally resonant material. Two geometries of the metallic frame will be considered: square shape, with edge $a$ (Fig. 1a) and hexagonal, honeycomb, shape, with edge $L$ (Fig. 1b). In the first case the lattice vectors are orthogonal, while in the second they are at $\pi / 3$. Figure 1 also shows the unit cell and the first Brillouin zone; $\mathbf{e}_{1}^{*}$ and $\mathbf{e}_{2}^{*}$ denote the reciprocal lattice vectors and the shaded area is the irreducible Brillouin zone (IBZ) which accounts for the symmetries. The vectors of the direct and reciprocal lattices are related by:

$$
\mathbf{e}_{i} \cdot \mathbf{e}_{j}^{*}=2 \pi \delta_{i j}
$$

In the numerical simulations we will consider the material constituents listed in Table 1.

Table 1: Elastic properties and density of the constituent materials

\begin{tabular}{c|c|c|c}
\hline Constituents & $E[\mathrm{MPa}]$ & $v$ & $\rho\left[\mathrm{kg} / \mathrm{m}^{3}\right]$ \\
\hline \hline Aluminum alloy & 70000 & 0.34 & 2600 \\
Material of the inclusion & 40800 & 0.37 & 11600 \\
Foam & 10 & 0.45 & 115 \\
\hline
\end{tabular}

If the out-of plane dimension $h$ is very large with respect to the periodicity length $a$ or $L$, it is common to consider separately in-plane polarized waves and out-of-plane polarized waves and to evaluate the dispersion diagrams for in-plane and out-of-plane modes. We will follow this approach in section 3.1 while in section 3.2 we will consider a full three-dimensional analysis.

\subsection{Decoupled in-plane and transversal analyses}

For the two-dimensional analysis of the cylindrical periodic solid, infinite in the out-of-plane direction, the wave vector $\mathbf{k}$ is confined to the $x-y$ plane and the modes are decoupled into in-plane modes, described by displacement 
vectors of the form $u_{x}(x, y) \mathbf{i}_{x}+u_{y}(x, y) \mathbf{i}_{y}$ and out-of-plane, pure transverse modes, described by displacement vectors of the form $u_{z}(x, y) \mathbf{i}_{z}$, with $\mathbf{i}_{x}, \mathbf{i}_{y}, \mathbf{i}_{z}$ denoting the unit vectors of the Cartesian reference frame. To determine the band structure the eigenvalue problem (3) is solved numerically for $\mathbf{k}$ belonging to the boundary $\Gamma-X-M-\Gamma$ of the IBZ. The reduced frequency $\omega a / 2 \pi$ of each mode is then plotted as a function of the arc length along the boundary $\Gamma-X-M-\Gamma$. The diagrams thus obtained are called dipersion diagrams and characterize the transmission properties of the LRM. The intervals of frequencies where no real solutions exist define the band-gaps.

The dispersion diagrams thus obtained are shown in Figs. 2 and 4 for the square and hexagonal lattices, different gray are used to distinguish the different modes. The two lattices have the same cell area i.e. $a^{2}=\frac{3 \sqrt{3}}{2} L^{2}$ and they are characterized by the same volume fraction of the aluminium frame (defined as cross area of the frame divided by the cross area of the cell) and of the lead inclusion (defined as cross area of the inclusion divided by the cross area of the cell); these volume fractions are equal to 0.15 and 0.44 , respectively. The dispersion plots for in-plane modes are obtained by plane strain analyses and, for the square and hexagonal lattice, they are reported in Figs $2 \mathrm{a}$ and $4 \mathrm{a}$, respectively, in terms of reduced frequency. Both are characterized by the presence of a band-gap, shaded in the figures, opening between the third and fourth mode.

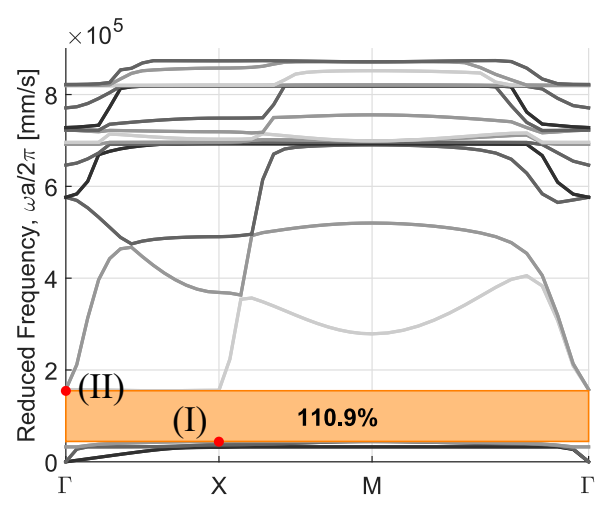

(a)

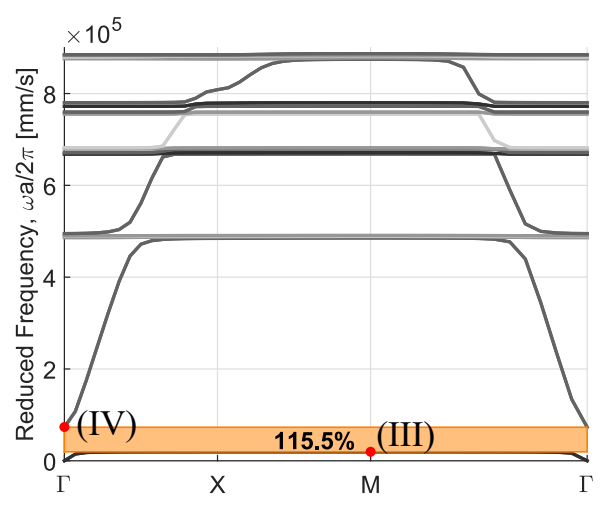

(b)

Figure 2: Band diagrams for 2D in-plane (a) and out-of-plane (b) modes in the LRM with square lattice and infinite value of $h$

The values of the opening and closing frequencies, corresponding to points 
I and II in Figs 2a and 4a, are reported in Table 2. The relative amplitude, defined as the bandwidth divided by the central band-gap frequency, is similar for the two lattices, $110.9 \%$ for the square lattice and $114.8 \%$ for the hexagonal one. For out-of planes modes the gap appears between the first and the second mode, see Figs $2 \mathrm{~b}$ and $4 \mathrm{~b}$.

Table 2: Opening, closing and central reduced frequencies $\omega a / 2 \pi$ of the first band-gap for the different configurations

\begin{tabular}{l|c|c|c} 
Unit cell & opening $[\mathrm{mm} / \mathrm{s}]$ & closing $[\mathrm{mm} / \mathrm{s}]$ & central $[\mathrm{mm} / \mathrm{s}]$ \\
\hline square in - plane & 44380. & 154872. & 99626. \\
square out - of - plane & 19580. & 73116. & 46348. \\
\hline hexagonal in - plane & 42077. & 155373. & 98725. \\
hexagonal out - of - plane & 18142. & 67750. & 42946. \\
\hline cubic open & 37643. & 117344. & 77494. \\
cubic walls & 42379. & 60523. & 51451. \\
cubic closed & 43055. & 97281. & 70168. \\
\hline prismatic open & 38589. & 108516. & 73553. \\
prismatic walls & 41669. & 60986. & 51328. \\
prismatic closed & 44888. & 117394. & 81141. \\
\hline
\end{tabular}

Figures 3 and 5 report the opening and closing modes for the square and hexagonal lattices that are marked by I, II, III and IV in Figures 2 and 4 , respectively. The opening and closing modes correspond to different symmetry points of the IBZ, as common for band-gaps generated by a local resonant mechanism. 


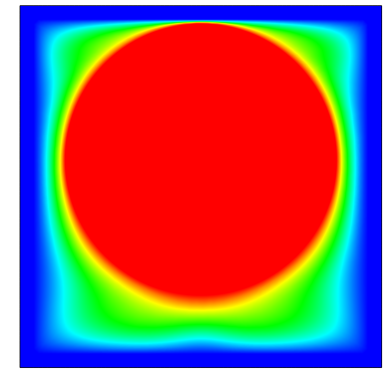

(I) Mode $3 \boldsymbol{k}$-point $X$

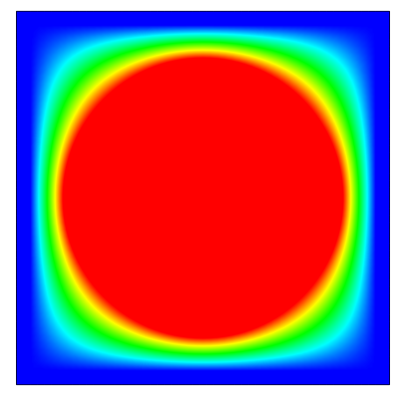

(III) Mode $1 \boldsymbol{k}$-point $M$

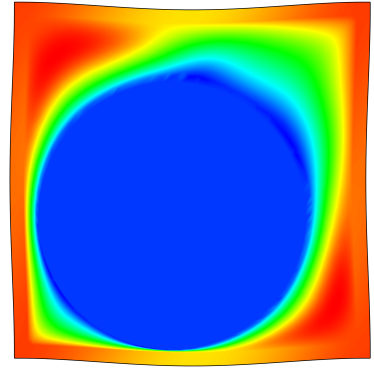

(II) Mode $4 \boldsymbol{k}$-point $\Gamma$
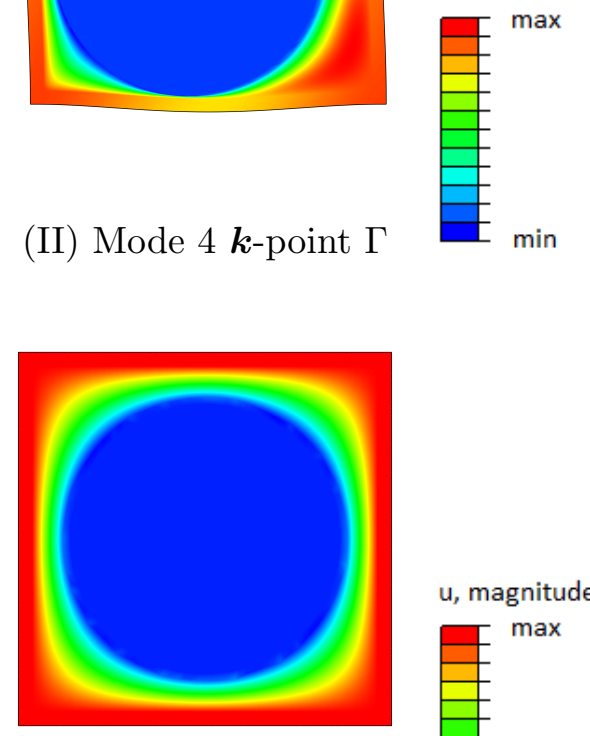

(IV) Mode $2 \boldsymbol{k}$-point $\Gamma$

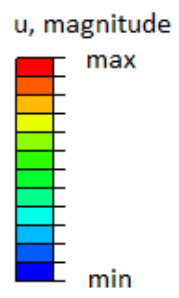

Figure 3: Opening and closing modes for the in-plane (I-II) and the out-of-plane (III-IV) analyses in the LRM with square lattice and infinite value of $h$ 


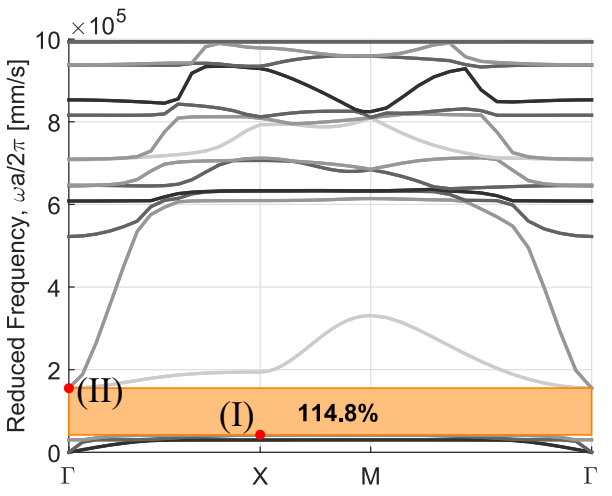

(a)

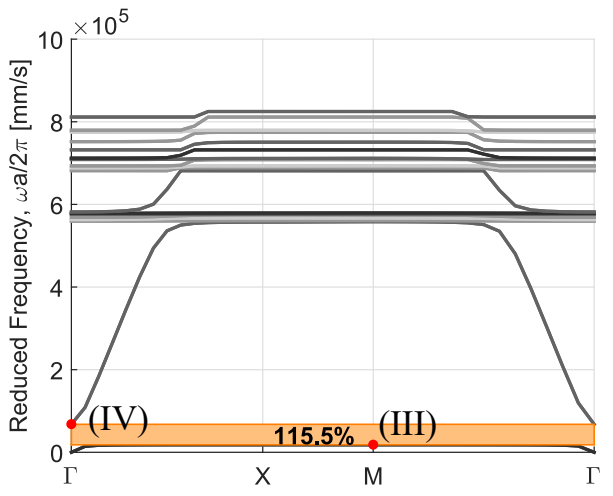

(b)

Figure 4: Band diagrams for: 2D in-plane (a) and out-of-plane (b) modes in the LRM with hexagonal lattice and infinite value of $h$

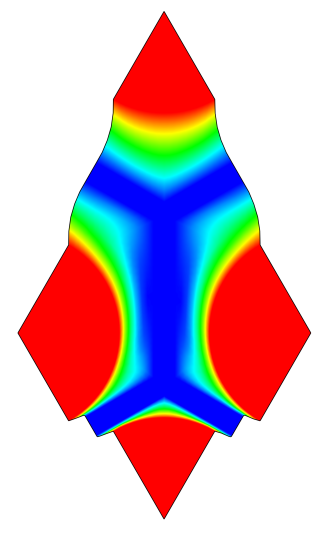

(I) Mode $3 \boldsymbol{k}$-point $X$

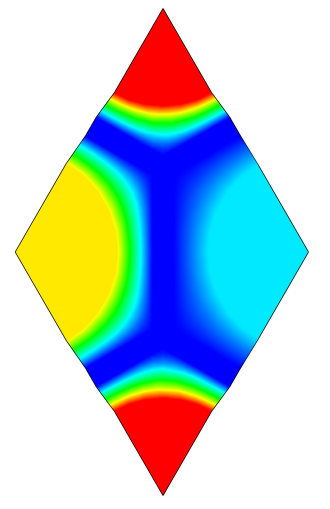

(III) Mode $1 \boldsymbol{k}$-point $M$

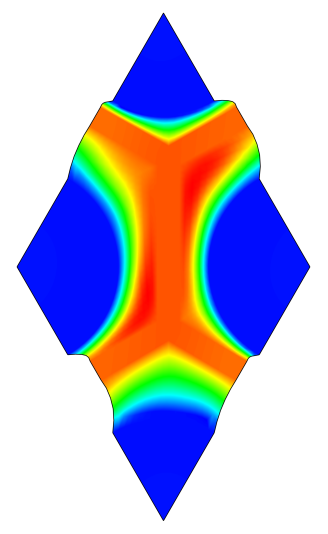

(II) Mode $4 \boldsymbol{k}$-point $\Gamma$ u, magnitude

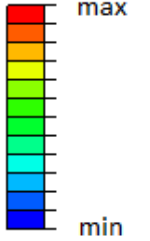

$u$, magnitude

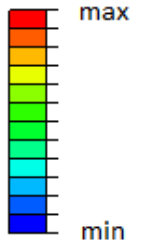

(IV) Mode $2 \boldsymbol{k}$-point $\Gamma$ 10

Figure 5: Opening and closing modes for the in-plane (I-II) and the out-of-plane (III-IV) analyses in the LRM with hexagonal lattice and infinite value of $h$ 
Keeping fixed the LRM components and the volumetric fraction of the frame and of the inclusion, the reduced frequency $\omega a / 2 \pi$ of the band-gap is fixed and one can easily shift the real frenquency by changing the size of the unit cell: a smaller lattice length leads to band-gaps at higher frequency. For the sake of comparison, we also consider 2D analyses in plane-stress conditions. The opening and closing modes are similar to those shown in Figure 3(I-II) and Figure 5(I-II), but the values of the corresponding frequencies are generally lower than the plane strain case: as an example, for the square lattice the opening and closing reduced frequencies are $32009 \mathrm{~mm} / \mathrm{s}$ and 107014 $\mathrm{mm} / \mathrm{s}$, respectively.

\subsection{Three-dimensional analysis}

To compute the band structure of a cylindrical LRM with a finite thickness $h$, one has to perform a three-dimensional eigenvalue analysis. However, being the periodicity of the material two-dimensional, the reciprocal lattice is also two-dimensional and the Block-Floquet conditions should be imposed only on the faces of the unit cell with normal laying in the $x-y$ plane. Therefore the IBZ is still that is shown in Figure 1 and also in the threedimensional dispersion analyses one can consider only wave vectors belong to the boundary $\Gamma, X, M, \Gamma$.

The band-gap position and amplitude change when changing the thickness $h$ of the material and the boundary conditions on the surfaces of normal $z$. Figure 6 shows the complete band-gaps obtained by full 3D analyses of prismatic unit cells with square cross section, free surfaces of normal $z$ and different out-of-plane thickness; the horizontal lines represent the limit of the band-gaps obtained for the in-plane and out-of-plane modes in the previous section for an infinite $h$. The intersection between these band-gaps represents the prediction of the complete band-gap, which in the present case has a relative magnitude of $48.9 \%$.

One can observe that the actual band-gap, represented by the blue bars in Figure 6, is well approximated by the two-dimensional analyses for $h \geq 2 a$, however, for very high values of the thickness, the band-gap structure changes since the frequency of some modes involving bending of the resonating cylinders decreases and enters into the band-gap. This phenomenon is evidenced in Figure 7 for $h=6 a$. The dispersion plot presents two smaller band-gaps separated by the $7^{\text {th }}$ and $8^{\text {th }}$ bending modes; these modes do not exist in the infinite solid. 


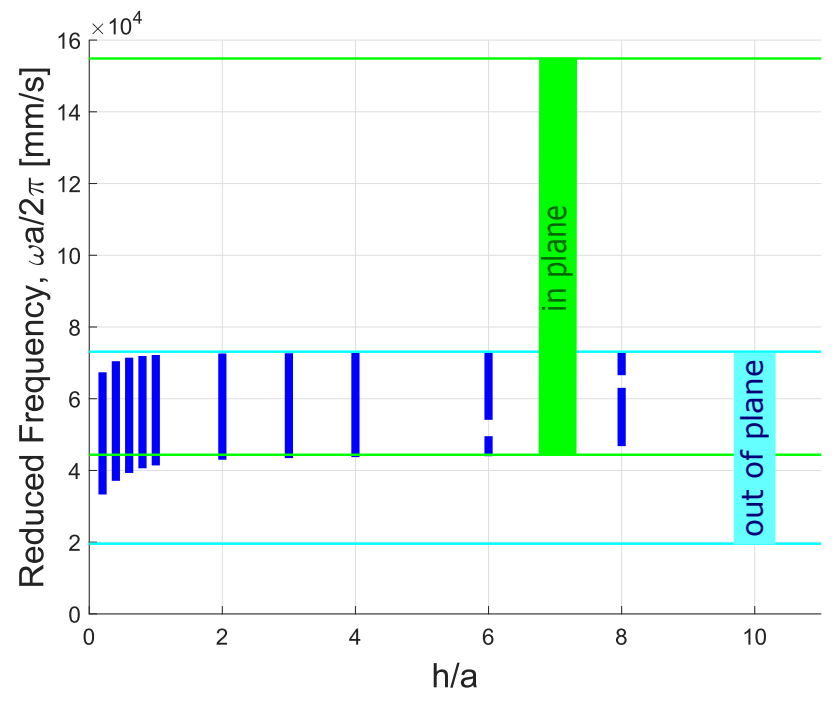

Figure 6: Complete 3D band diagram in the LRM with square lattice for varying $h$ and free end surfaces

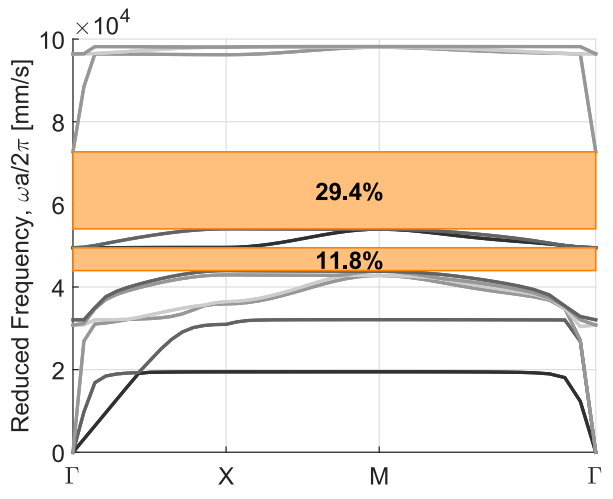

(a)

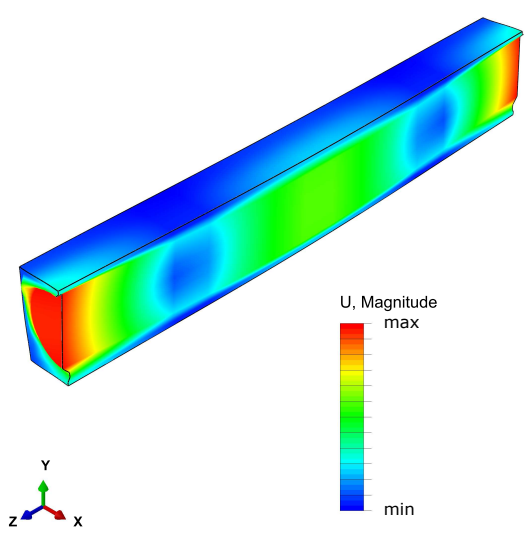

(b)

Figure 7: Cylindrical LRM with square lattice, $h=6 a$ and free end surfaces: (a) dispersion diagram with two separated band-gaps, (b) $7^{\text {th }}$ bending mode at the $\mathbf{k}$ point $\Gamma$ (only half of the cell is shown for representation clarity). 
If the solid has restrained end surfaces (in $z$-direction), the out-of-plane modes are inhibited and one obtains a different band stucture, as shown in Figure 8 for the case $h=4 a$. Note that there is the formation of four band-gaps all inside the frequence interval defined by the band-gap under plane-strain conditions. There are some modes, characterized by non uniform displacements in the $z$-direction, that enter into the bandgap of the ideal plane-strain case which is only valid for a solid infinite in $z$.

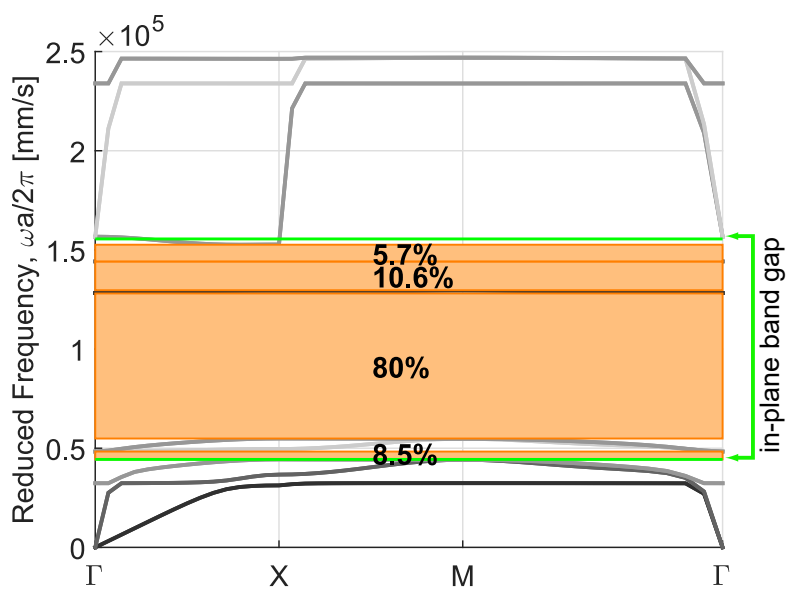

Figure 8: Cylindrical LRM with square lattice, $h=4 a$ and fixed end surfaces: dispersion diagram with four separated band-gaps within the bandgap limits for the square $2 \mathrm{D}$ in-plane case (in green).

It is interesting to compare the results of $3 \mathrm{D}$ analyses with the $2 \mathrm{D}$ plane stress case. The opening frequency coincides with that obtained with $3 \mathrm{D}$ analyses with free BCs in the z-direction, in the case of small h/a. This confirms the theoretical prediction that plane stress analyses are well suited for thin solids. On the other hand, the intersection of in-plane (plane stress) and out-of-plane results does not match the 3D bandgap, in view of the fact that the decoupled analyses cannot predict the closing mode for small h/a. Such a mode is characterized by out-of-plane motion that, owing to the small thickness, involves some local bending of the cell. The decoupled analyses are not able to include bending behavior.

From the above analyses we can conclude that: $(i)$ decoupled plane strain/outof-plane analyses can provide a good estimate of the bandgap for large $\mathrm{h} / \mathrm{a}$; (ii) in the case of small $\mathrm{h} / \mathrm{a}$, plane stress analysis can be used to predict the 
opening frequency, but the closing one can be obtained only with a coupled 3D analysis.

Similar results are obtained for the hexagonal lattice as shown in Figure 9. For $2 L<h<8 L$ the first complete band-gap coincides with the one obtained as intersection of the band-gaps for in-plane and out-of-plane waves; for lower values of $h$ the complete band-gap is shifted to lower frequencies, while for $h \geq 8 L$ the first band-gap is interrupted by a flexural mode, similar to the one shown in Figure 7b.

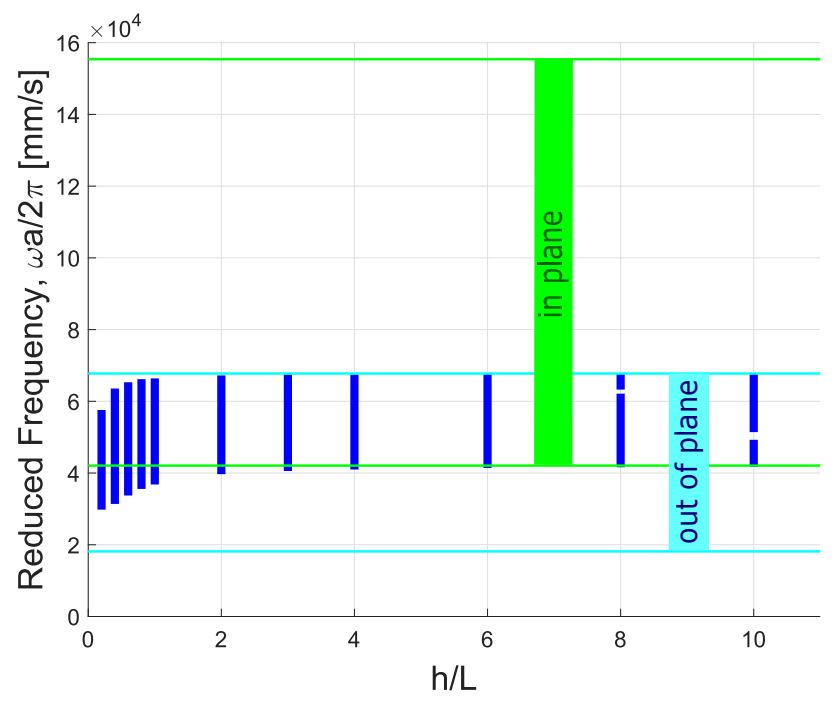

Figure 9: Complete 3D band-gap in the cylindrical LRM with hexagonal lattice for varying $h$ and free end surfaces

\section{Band-gap in three-dimensional locally resonant material}

In this section we consider locally resonant materials endowed with threedimensional periodicity, we refer to these materials as 3D-LRM in contrast with the cylindrical-LRM considered above. The unit cell, which periodically repeats in the three directions, can have a cubic shape of edge $a$ (see Figure 10a,b,c) or a prismatic one with $L=1.0825 a$ (see Figure 10d,e,f). The material has the same constituents listed in Table 1; the inclusion is a heavy sphere of radius $R=\frac{3}{8} a$, immersed in a polymeric foam matrix, embedded in aluminium structures with different geometries. Three configurations of the aluminium structure are explored as shown in Figures 10: (a, d) cellular 
cylindrical structure such that the cell has lateral walls only, (b, e) closed structure with walls also in the third direction, (c, f) open frame with beam elements at each edge. In all cases the thickness of the aluminium structure is $t=0.04 a$. Configurations (a) and (d) are simpler from a technological point of view, since the aluminium structure can be extruded and the only difference with the cylindrical-LRM is that the inclusions are spheres instead of cylinders.

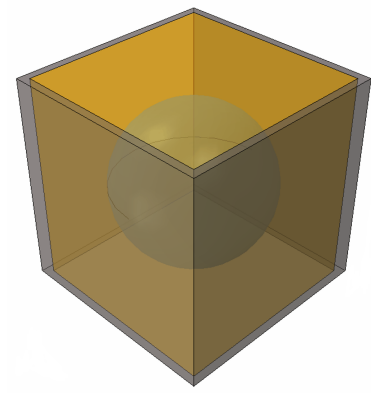

(a)

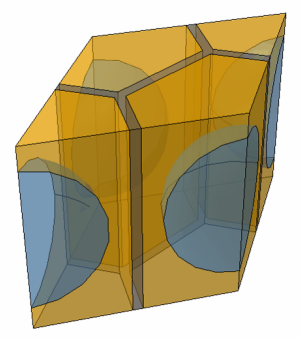

(d)

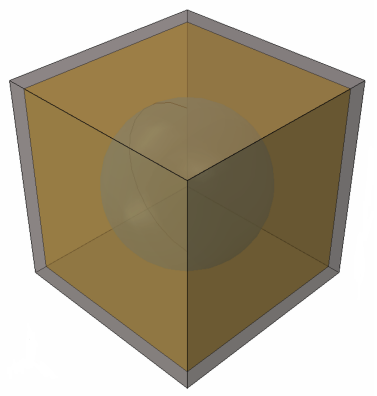

(b)

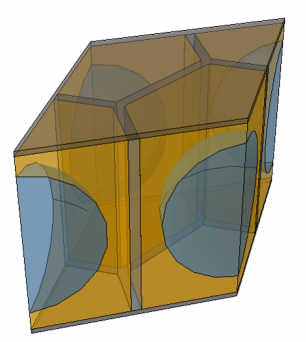

(e)

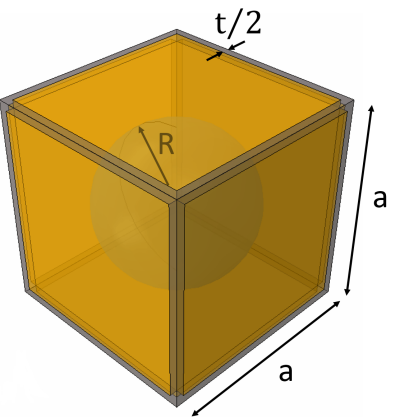

(c)

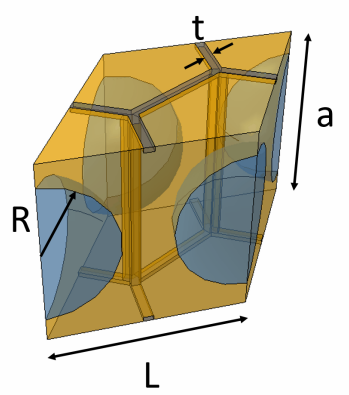

(f)

Figure 10: Unit cells for the cubic crystals: (a) lateral walls, (b) closed cell, (c) open frame only; unit cells for the prismatic-hexagonal crystals: (d) lateral walls, (e) closed cell, (f) open frame only

Let us first consider the lattice with cubic cells; being the periodicity 3D, the reciprocal lattice is also $3 \mathrm{D}$ and the first Brillouin zone is a cube with orthogonal reciprocal lattice vectors (Figure 11a). Considering the symmetries, one can restrict the analysis to the tetrahedron $\Gamma-X-R-M$ which 
constitutes the IBZ

The dispersion analysis is performed by solving the eigenvalue problem (3) numerically, with $\mathbf{k}$ belonging to the boundary of the IBZ. The resulting dispersion plots for the cubic cells are shown in Figures 11b-d. On Figure 11b, I and II indicate the opening and closing points of the first band-gap, similarly on Figure 11c, III and IV indicate the opening and closing points; the corresponding modes are shown in Figures 12 I, II, III and IV, respectively. The values of the opening, closing and central band-gap reduced frequencies are also reported in Table 2 .

One can observe that the configuration with four lateral walls leads to the smaller band-gap among the three proposed geometries of 3D-LRM. Furthermore, the relative magnitude of the band-gap (35.3\%) is lower that the magnitude of the complete band-gap (48.9\%) obtained with the corresponding cylindrical-LRM. This is due to the lower mass of the resonating part (a sphere instead of a cylinder of equal radius).

The use of a completely closed cell allows, instead, to widen the ban-gap (Figure 11c) as it increases the frequency of the closure mode. Actually, as shown in Figure 12, while the opening modes are similar for the two cells (cp. Figures 12 I and III), the closure mode for the cell with only lateral walls (Figures 12 II) involves a vibration of the inclusion in the third direction; if the cell is completely closed this mode cannot occur, the closure mode changes (see Figure 12 IV) and a wider band-gap is obtained.

The widest band-gap is obtained for the cell with an open aluminium frame (Figure 11c) as in this case the ratio between the resonating mass and the total mass is maximum. This is in agreement with results reported in the literature for other LRMs [28]. 


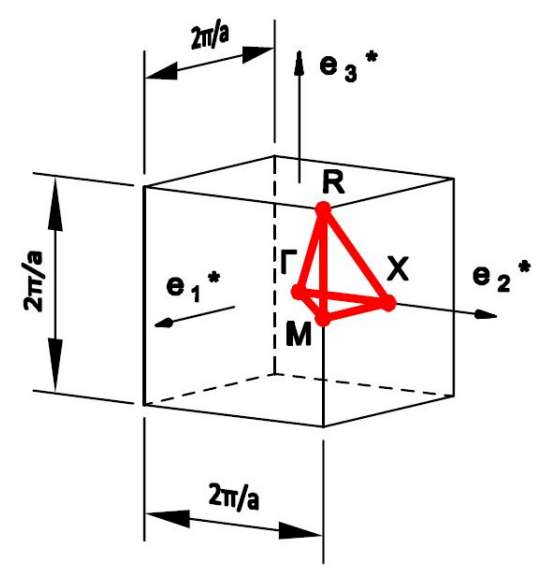

(a)

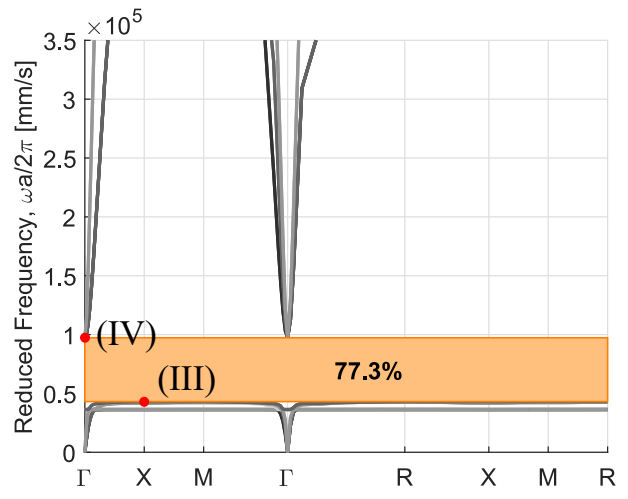

(c)

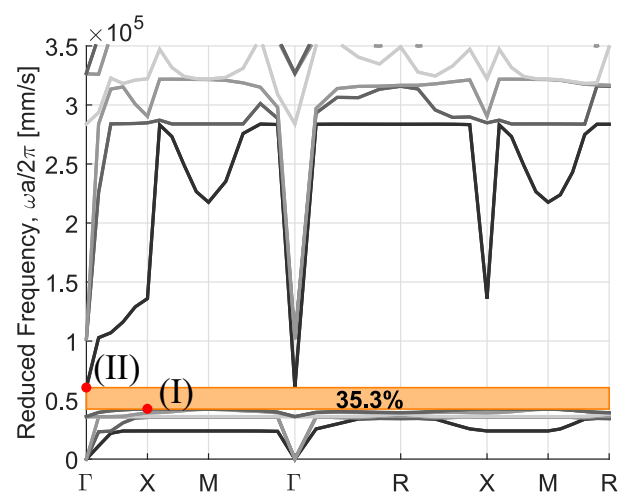

(b)

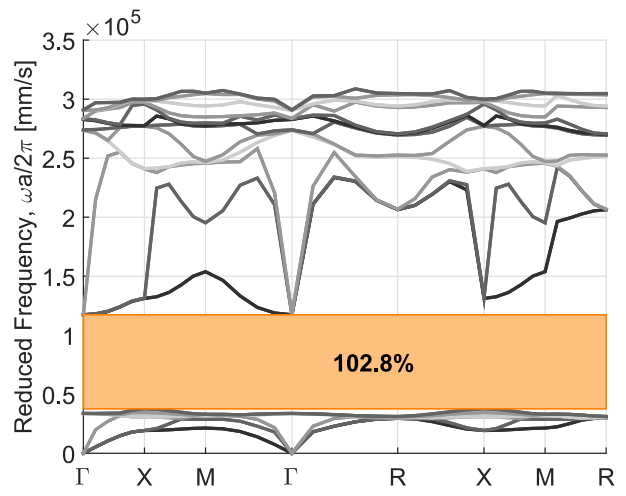

(d)

Figure 11: 3D three-components LRMs with cubic lattices: (a) first Brillouin zone and IBZ; band diagrams for (b) cell with lateral walls, (c) closed cell, (d) open frame only. 


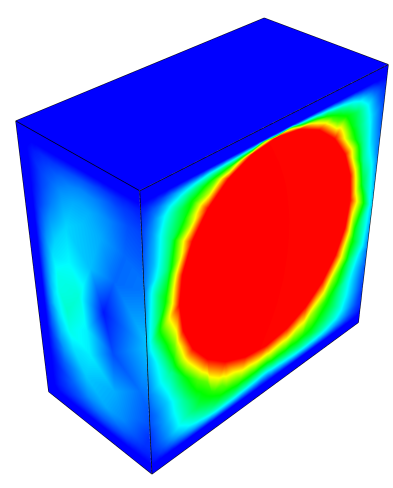

(I) Mode $6 \boldsymbol{k}$-point $X$

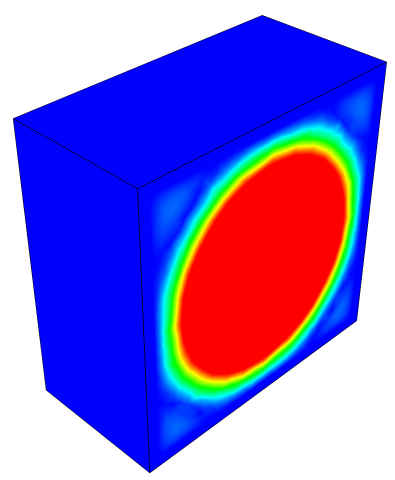

(III) Mode $6 \boldsymbol{k}$-point $X$

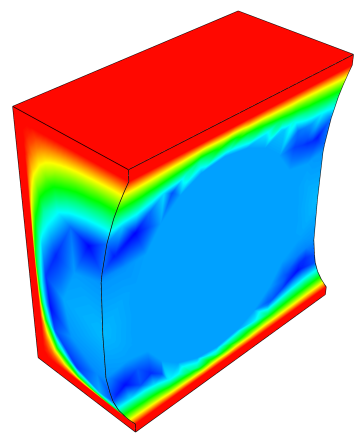

(II) Mode $7 \boldsymbol{k}$-point $\Gamma$
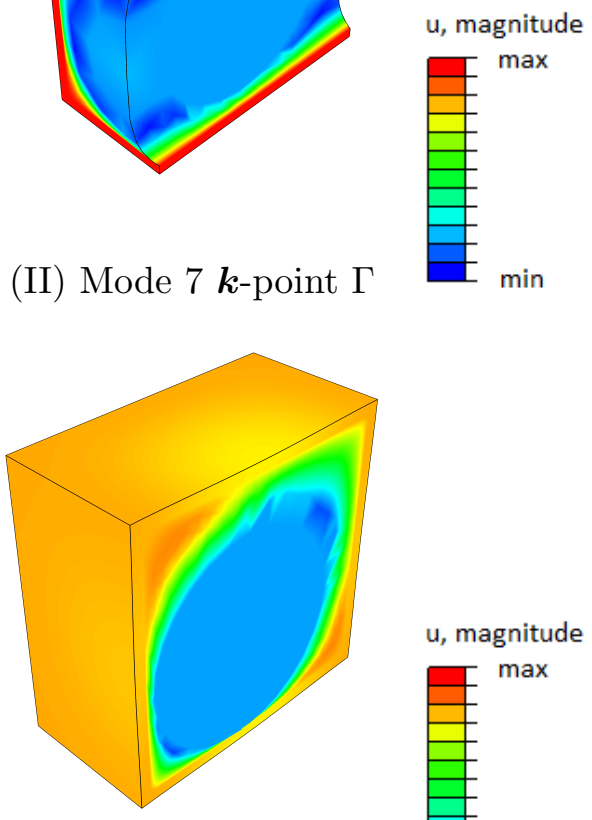

(IV) Mode $7 \boldsymbol{k}$-point $\Gamma$

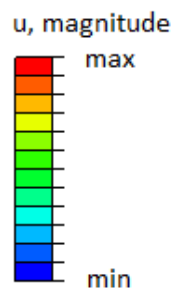

Figure 12: Relevant modes for the 3D cubic crystals (I) opening mode for the cell with lateral walls, (II) closure mode for the cell with lateral walls, (III) opening mode for the closed cell, (IV) closure mode for the closed cell.

We consider now the hexagonal-prismatic lattice: in this case the first Brillouin zone is also a prism with hexagonal base, Figure 13a. Exploiting the symmetries, the analysis can be restricted to the IBZ which is the triangular prism $\Gamma-X-M-A-L-H$.

The band diagrams for the three cells with different aluminium structures are shown in Figures 13b-d. As in the case of a cubic lattice, the widest band-gap is obtained for the case of an open aluminium frame (d), while the configuration with an extruded aluminium structure (b) leads to the smallest band-gap. 


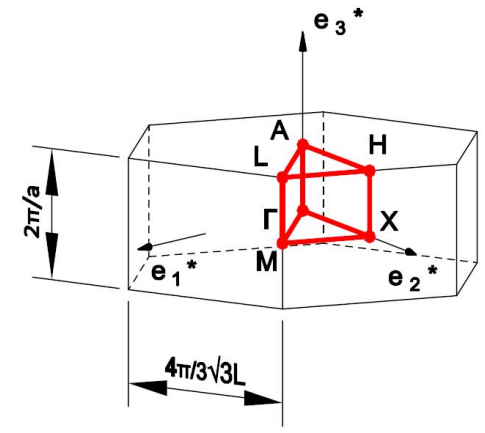

(a)

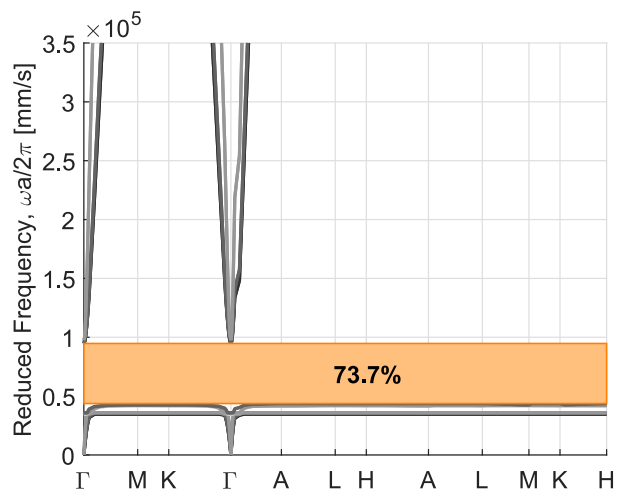

(c)

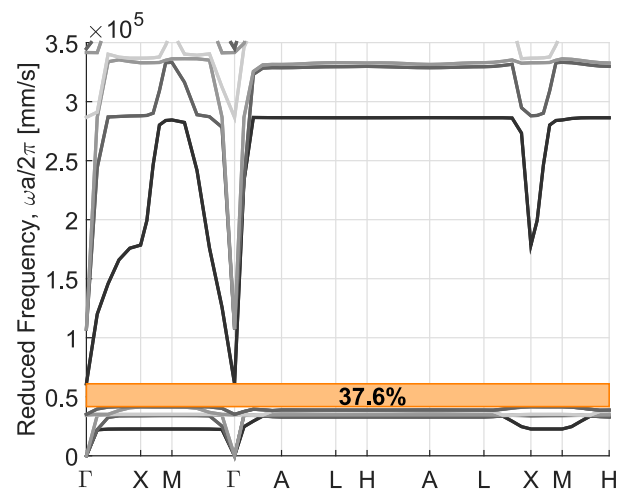

(b)

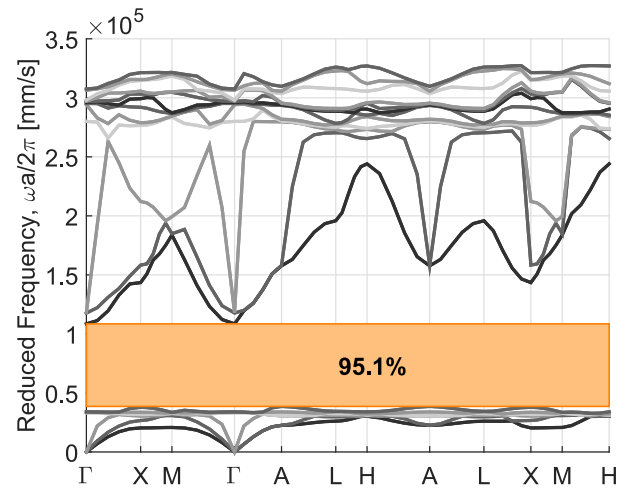

(d)

Figure 13: 3D three-components LRMs with prismatic-hexagonal lattices: (a) first Brillouin zone and IBZ; band diagrams for (b) cell with lateral walls, (c) closed cell, (d) open frame only.

\section{Conclusions}

This paper investigates the dispersion properties of cellular locally resonant materials, with different geometries, through finite element modal analyses conducted on the unit cell with imposed Bloch-Floquet boundary conditions.

For LRM with 2D periodicity (or cylindrical LRM) the band structure calculated separately for in-plane and out-of-plane modes is compared with that obtained by a three-dimensional analysis on solids with different out-of plane 
thickness and boundary conditions. It is shown that, considering free boundary conditions, the real band structure depends on the out-of-plane thickness of the material and it can be smaller than that of the ideal infinite material, evaluated as the intersection of those obtained by decoupled 2D analyses, because some bending modes can enter into the stop band. On the contrary, the width of the complete band-gap can be increased by designing LRM with 3D periodicity, with spherical inclusions. In particular, when the periodic cell is open, with a stiff frame only, the gap-mid gap ratio reaches the unit.

These results open the way to the design of new three-components cellular LRM to be used for vibration isolation or energy absorption. However, it must be noted that the comparative considerations are based on the dispersion analyses, in the hypothesis of linear elastic behavior. The effectiveness of LRM for specific applications must be assessed by taking into account also the possible non-linear phenomena connected to the peculiar loading conditions. More specifically, the elastic buckling of frame elements or the occurrence of plastic strain in the material components might affect the actual transmission properties of the LRM. The results presented in this paper could represent a guideline to ideate LRM with optimal performances in terms of bandgap in the frequency spectrum, but the final design must be accompanied by trasmission analyses, as well as safety assessment in the ultimate and serviceability limit states.

Acknowledgments - Financial support of the Italian MIUR (PRIN project nr.2015LYYXA8 on Multi-scale mechanical models for the design and optimization of micro-structured smart materials and metamaterials) and of EU (Erasmus Mundus Programme Smart ${ }^{2}$ ) is gratelully acknowledged.

\section{References}

[1] Z. Liu, X. Zhang, Y. Mao, Y. Y. Zhu, Z. Yang, C. T. Chan, P. Sheng, Locally resonant sonic materials, Science 289 (5485) (2000) 1734-1736.

[2] T. Gorishnyy, M. Maldovan, C. Ullal, E. Thomas, Sound ideas, Physics World 18 (12) (2005) 24-29.

[3] M. Maldovan, Sound and heat revolutions in phononics, Nature 503 (7475) (2013) 209-217.

[4] G. Ma, P. Sheng, Acoustic metamaterials: From local resonances to broad horizons., Science Advances 2 (2) (2016) e1501595. 
[5] T. Delpero, S. Schoenwald, A. Zemp, A. Bergamini, Structural engineering of three-dimensional phononic crystals, Journal of Sound and Vibration 363 (2015) 156-165.

[6] L. D'Alessandro, E. Belloni, R. Ardito, A. Corigliano, F. Braghin, Modeling and experimental verification of an ultra-wide bandgap in 3D phononic crystal, Applied Physics Letters 109 (22) (2016) 221907.

[7] L. D’Alessandro, E. Belloni, R. Ardito, F. Braghin, A. Corigliano, Mechanical low-frequency filter via modes separation in 3D periodic structures, Applied Physics Letters 111 (23) (2017) 231902.

[8] O. Bilal, M. Hussein, Ultrawide phononic band gap for combined inplane and out-of-plane waves, Physical Review E - Statistical, Nonlinear, and Soft Matter Physics 84 (6) (2011) 065701.

[9] P. Wang, J. Shim, K. Bertoldi, Effects of geometric and material nonlinearities on tunable band gaps and low-frequency directionality of phononic crystals, Physical Review B - Condensed Matter and Materials Physics 88 (1) (2013) 014304.

[10] L. D'Alessandro, V. Zega, R. Ardito, A. Corigliano, 3D auxetic single material periodic structure with ultra-wide tunable bandgap, Scientific Reports 8 (1) (2018) 2262.

[11] Y. Chen, G. Hu, G. Huang, A hybrid elastic metamaterial with negative mass density and tunable bending stiffness, Journal of the Mechanics and Physics of Solids (105) (2017) 179-198.

[12] A. Bacigalupo, M. Lepidi, G. Gnecco, L. Gambarotta, Optimal design of auxetic hexachiral metamaterials with local resonators, Smart Materials and Structures 25 (5) (2016) 054009.

[13] L. D’Alessandro, B. Bahr, L. Daniel, D. Weinstein, R. Ardito, Shape optimization of solidair porous phononic crystal slabs with widest full 3D bandgap for in-plane acoustic waves, Journal of Computational Physics 344 (2017) 465-484.

[14] V. Laude, Phononic Crystals. Artificial Crystals for Sonic, Acoustic, and Elastic Waves, Berlin, Boston: De Gruyter, 2015. 
[15] C. Croënne, E. Lee, H. Hu, J. Page, Band gaps in phononic crystals: Generation mechanisms and interaction effects, AIP Advances 1 (2011) 041401-1.

[16] G. W. Milton, J. R. Willis, On modifications of Newton's second law and linear continuum elastodynamics, Proceedings of the Royal Society A 463 (January) (2007) 855-880.

[17] X. Zhou, X. Liu, G. Hu, Elastic metamaterials with local resonances: an overview, Theoretical and Applied Mechanics Letters 2 (4) (2012) 041001.

[18] M. V. Barnhart, X. Xu, Y. Chen, S. Zhang, J. Song, G. Huang, Experimental demonstration of a dissipative multi-resonator metamaterial for broadband elastic wave attenuation, Journal of Sound and Vibration (438) (2019) 1-12.

[19] X. Xu, M. V. Barnhart, X. Li, Y. Chen, G. Huang, Tailoring vibration suppression bands with hierarchical metamaterials containing local resonators, Journal of Sound and Vibration (442) (2019) 237-248.

[20] A. O. Krushynska, V. G. Kouznetsova, M. G. D. Geers, Towards optimal design of locally resonant acoustic metamaterials, Journal of the Mechanics and Physics of Solids 71 (1) (2014) 179-196.

[21] K. T. Tan, H. H. Huang, C. T. Sun, Blast-wave impact mitigation using negative effective mass density concept of elastic metamaterials, International Journal of Impact Engineering 64 (2014) 20-29.

[22] D. Briccola, M. Ortiz, A. Pandolfi, Experimental validation of metaconcrete blast mitigation properties, Journal of Applied Mechanics, Transactions ASME 84 (3) (2017) 031001.

[23] C. Comi, L. Driemeier, Metamaterials for crashworthiness of small cars, in: AIMETA 2017, Salerno, Italy, 2017, pp. 2119-2129.

[24] C. Comi, L. Driemeier, Wave propagation in cellular locally resonant metamaterials, Latin American Journal of Solids and Structures 15 (4) (2018) e38. 
[25] A. O. Krushynska, M. Miniaci, V. G. Kouznetsova, M. G. D. Geers, Multilayered inclusions in locally resonant metamaterials: two-dimensional versus three-dimensional modeling, Journal of Vibration and Acoustics 139 (2017) 024501-1-4.

[26] C. Kittel, Elementary Solid State Physics: A Short Course, 1st Edition, New York, Wiley, 1962.

[27] A. S. Phani, J. Woodhouse, N. A. Fleck, Wave propagation in twodimensional periodic lattices, J. Acoust. Soc. Am. 119 (4) (2006) 19952005 .

[28] L. D'Alessandro, E. Belloni, G. D'Al, L. Daniel, R. Ardito, A. Corigliano, F. Braghin, Modelling and experimental verification of a single phase three-dimensional lightweight locally resonant elastic metamaterial with complete low frequency bandgap, in: Metamaterials 2017, 2017, pp. $70-72$. 